\title{
Coupling with two-center neutron states and two-surface collective excitations at fusion reactions in the vicinity of the Coulomb barrier
}

\author{
Viacheslav Samarin \\ Flerov Laboratory of Nuclear Reactions, JINR, 141980 Dubna, Moscow Region, Russia
}

\begin{abstract}
A clear physical interpretation of barrier distribution peaks and depressions is proposed. It is based on distance dependent vibration excitation energies near the barrier. Microscopic coupled channel equations with neutron rearrangement coupling were proposed and solved. They used distance dependent Q-values near the barrier. The satisfactory agreement between experimental data and calculation results is obtained for fusion cross sections in two reactions ${ }^{18} \mathrm{O}+{ }^{58} \mathrm{Ni},{ }^{16} \mathrm{O}+{ }^{60} \mathrm{Ni}$ in the vicinity of the Coulomb barrier.
\end{abstract}

\section{Introduction}

Two interesting aspects of fusion reactions are the fine structure of the barrier distribution function and the increase of fusion cross sections in reactions with some neutron-rich nuclei. The well known [1-3] coupled channel method is evolved to include these aspects in the microscopic description by solving two-center and timedependent Schrödinger equations.

\section{The vibration channels coupling}

The vibration channel coupling effects in fusion reactions were studied using coupled channel equations [1-3]

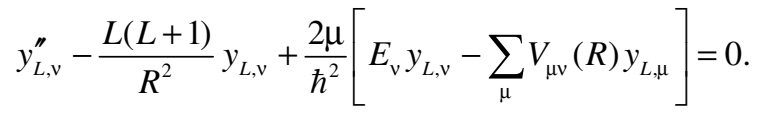

Here $L$ is the orbital momentum of partial wave, is the reduced mass, $E=E_{\text {c.m. }} \quad, E_{\text {c.m. }}=\hbar^{2} k_{0}^{2} / 2$ is the energy in the centre of mass system, is the nuclear excitation energy in the channel , $V(R)=\langle|V(R, \boldsymbol{\beta})|\rangle \quad$ is the coupling matrix, I ) ( $\boldsymbol{\beta})$ are harmonic oscillator functions, $\boldsymbol{\beta}=\left\{\begin{array}{c}i \\ i\end{array}\right\}$, is the deformation parameter multipolarity ; $V(R, \boldsymbol{\beta})$ is the nucleus-nucleus interaction [3], $R$ is the internuclear distance. An approximate expression for the function $V(R, \boldsymbol{\beta})$ with the proximity potential was obtained in [3]. The well-known CCFULL code [2] (for Woods-Saxon potential) and the code included in the NRV scientific web server [4] (for Woods-Saxon and proximity potentials) are often used for the fusion cross section calculation (Fig. $1 a$ )

$$
\begin{aligned}
\text { fus } & =\frac{\hbar^{2}}{2 E_{\text {c.m. }} j_{0}} \sum_{L=0}(2 L+1) \sum\left|j_{L, v}\right|, \\
j_{L v} & =\left.i \frac{\hbar}{2}\left(y_{L v} \frac{d y_{L}^{*}}{d R} \quad y_{L}^{*} \frac{d y_{L v}}{d R}\right)\right|_{R R_{1}+R_{2}} .
\end{aligned}
$$

Here $j_{L v}$ is the incoming fusion flux to the compound nucleus region in the channel and $j_{0}=\hbar k_{0} /$.
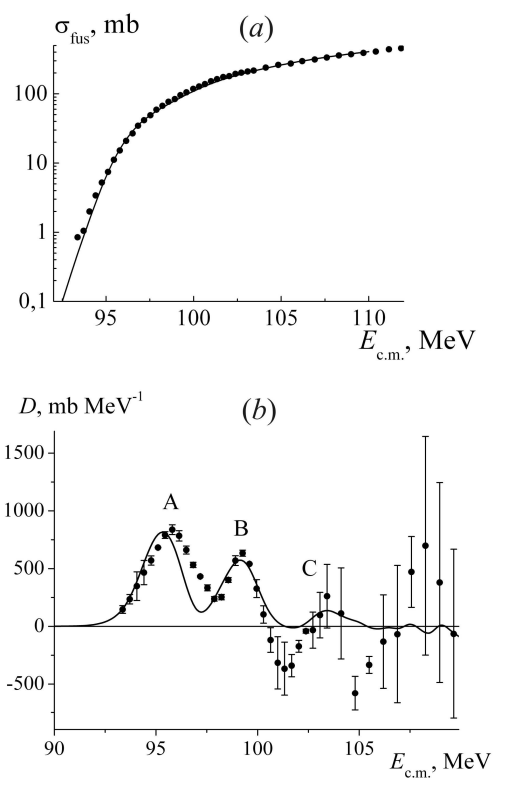

Figure 1. (a) Experimental data [5] (points) and the calculated by formula (2) (the solid curve) fusion cross section $\left(E_{\text {c.m. }}\right)$ of reaction ${ }^{40} \mathrm{Ca}+{ }^{90} \mathrm{Zr}$. (b) The barrier distribution $D\left(E_{\text {c.m. }}\right)$ extracted from this experimental data by spline fitting (points) and calculated by formulas (2), (3) (the solid curve).

\footnotetext{
${ }^{\mathrm{a}}$ Corresponding author: samarin@jinr.ru
} 
The experimental fusion cross section fus $\left(E_{\text {c.m. }}\right)$ may have a fine structure of the barrier distribution function $D\left(E_{\text {c.m. }}\right)$ (see Fig. $\left.1 b\right)$

$$
D\left(E_{\text {c.m. }}\right)=d^{2}\left(E_{\text {c.m. }} \text { fus }\right) / d E_{\text {c.m. }}^{2} \text {. }
$$

The function $D(E)$ is ambiguously determined because of experimental errors. To obtain $D(E)$ we used the mathematically correct procedure of the two-stage spline smoothing. In the initial stage the smoothing function $f(E)=\ln (F(E)), F(E)=E$ fus $(E)$ was found from the condition of the minimum of the functional [6]

$$
{ }_{1}[f(E)]=\int_{E_{0}}^{E_{n}}[f(E)]^{2} d E+\sum_{k=0}^{n} p_{k}{ }^{1}\left[f\left(E_{k}\right) \quad f_{k}\right]^{2},
$$

with experimental values $f_{k}=\ln \left(\begin{array}{ll}E_{k} & \text { fus }, k\end{array}\right)$. The values $p_{k}$ were found from the conditions

$$
\left|f\left(E_{k}\right) \quad f_{k}\right| \quad \text { fus } / \text { fus } .
$$

The barrier distribution function values

$$
D_{k}=F\left(E_{k}\right)=g\left(E_{k}\right) \exp \left(g\left(E_{k}\right)\right),
$$

and estimations of their errors

$$
D_{k}=\left|g\left(E_{k}\right) \exp \left(g\left(E_{k}\right)\right) \quad g_{0}\left(E_{k}\right) \exp \left(g_{0}\left(E_{k}\right)\right)\right|,
$$

were calculated on the second stage by the smoothing function

$$
g(E)=\ln F(E)=f(E)+\ln f(E) .
$$

It was found from the condition of the minimum of the functional analogous to (5). The function $g_{0}(E)$ is the result of the calculation without smoothing at the limit $p_{k} \quad 0$. The function $D\left(E_{\text {c.m. }}\right)$ for the reaction ${ }^{40} \mathrm{Ca}+{ }^{90} \mathrm{Zr}$ and data from [5] is shown in Fig. $1 b$.

The partial probability density $\left|{ }_{L}(R,)\right|^{2}$,

$$
{ }_{L}(R, \boldsymbol{\beta})=\sum y_{L,} \mid>\text {, }
$$

for the reaction ${ }^{40} \mathrm{Ca}+{ }^{90} \mathrm{Zr}, L=0$ is shown in Fig. 2. For the energy $E_{\text {c.m. }}=96 \mathrm{MeV}$ near the first peak A in Fig. $1 b$ the partial probability density has one jet across the multi-dimensional potential barrier $V\left(R,{ }_{23}\right)$ (Fig. $2 a$ ). It is similar to the ground deformation vibrational state. For the energy $E_{\mathrm{c} . \mathrm{m} .}=100 \mathrm{MeV}$ near the second peak B in Fig. $1 b$ the wave function ${ }_{0}(R$,$) has two jets$ across the multi-dimension potential barrier (Fig. $2 b$ ). It is similar to the first excited deformation vibrational state. Therefore the barrier distribution may be interpreted using the energy levels $(R)$ of the twosurface quadrupole and octupole vibrations of nuclei closely located at the distance $R$ [7].

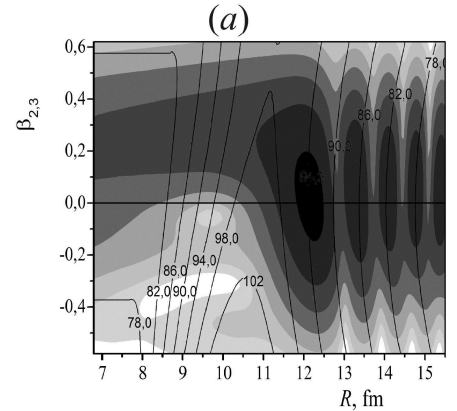

(b)

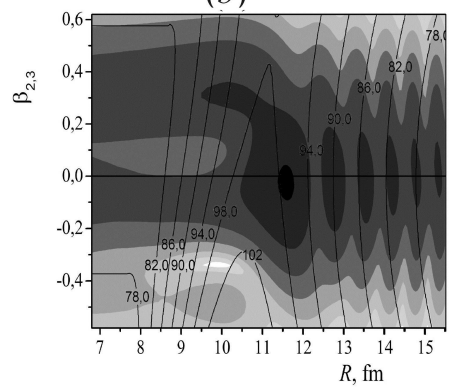

Figure 2. The probability density $\left|\left(R,{ }_{23}\right)\right|^{2}$ flow across the two-dimensional potential barrier $V\left(R,{ }_{23}\right)$ for the reaction ${ }^{40} \mathrm{Ca}+{ }^{90} \mathrm{Zr}, L=0$ and energy $E_{\text {c.m. }}$ equal to $96 \mathrm{MeV}(a)$ and $100 \mathrm{MeV}(b) ; R$ is the internuclear distance; ${ }_{23}$ is the octupole deformation parameter of the ${ }^{90} \mathrm{Zr}$ nucleus; values of $V$ are shown.

Energies $(R)$ and wave functions of excited stationary two-surface vibration states can be found from the Schrödinger equation

$$
\left[\sum_{i} H_{i}+V(R, \boldsymbol{\beta}) \quad V(R, 0)\right] \quad(R, \boldsymbol{\beta})=(R)
$$

Where $H_{i}$ is the Hamiltonian of the independent vibration of the $i$ th $(i=1,2)$ nucleus surface, that has a multipolarity and ${ }_{0}(\quad)=0$. In order to solve approximately the problem specified by Eq. (11), we use an expansion in harmonic-oscillator functions. In just the same way as in the coupled channel method [3], we take into account quadrupole and octupole vibrations of both the nuclei, with numbers $n_{1}$ and $n_{2}$ of corresponding phonons satisfying the amplitude and energy limitation. The total vibration energy of each nucleus is limited to

$$
n_{2 i \quad 2 i}+n_{3 i \quad 3 i}<\quad \max , i_{i}, n=0,1, \ldots, i=1,2 .
$$

Here $\max , i_{i}$ is the upper limit for energies of vibration excitations that corresponds to their mixing with closely lying non-collective excitations. We used the estimate of the upper limit ${ }_{\max } \sim{ }_{\mathrm{s}} / 2$, where $\mathrm{s}$ is the neutron separation energy. Such calculations by the coupledchannel method with the proximity potential for the ${ }^{40} \mathrm{Ca}+{ }^{90} \mathrm{Zr}$ fusion led to satisfactory agreement with the experimental data on the cross section $\left(E_{\mathrm{c} . \mathrm{m} .}\right)$ and on the barrier distribution $D\left(E_{\mathrm{c} . \mathrm{m} .}\right)$ (Fig. 1). Calculated energies $(R)$ of modified two-surface vibration states 
in the ${ }^{40} \mathrm{Ca}+{ }^{90} \mathrm{Zr}$ systems are plotted in Fig. 3. In the vicinity of the Coulomb barrier (at a distance $R=R_{\mathrm{B}}$ ) the decrease of energies $(R)<$ ( ) accounts for extension deformations of nuclei meeting one another. The spacings between neighbouring vibrational levels depend on $R$; therefore, in general, excitation energies in the barrier region differ from respective excitation energies in isolated nuclei.

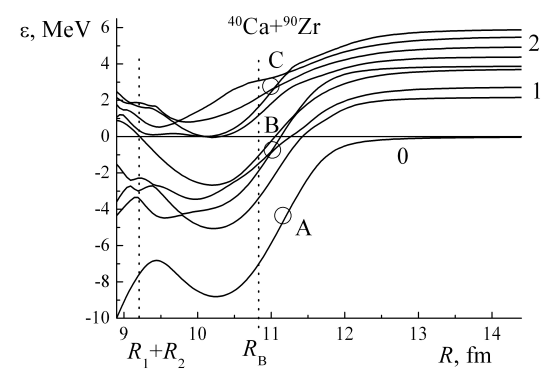

Figure 3. Energies $\varepsilon(R)$ of two-surface quadrupole and octupole vibration levels in the ${ }^{40} \mathrm{Ca}+{ }^{90} \mathrm{Zr}$ system: the ground state (the curve 0 ), excited states going over to single- and two-phonon states of ${ }^{90} \mathrm{Zr}$ nuclei for $R \quad$ (curves 1 and 2, respectively). Points $\mathrm{A}, \mathrm{B}$, and $\mathrm{C}$ correspond to most populated states before the barriers of adiabatic potentials (14), $R_{\mathrm{B}}$ is the radius of the Coulomb barrier top for spherical nuclei of radii $R_{1}$ and $R_{2}$.

In the adiabatic approximation, the potential energy of the nuclear interaction for the modified vibration state with number can be characterized by the effective potential

$$
V(R)=V(R)+\quad(R), \quad=0,1, \ldots,
$$

and by the potential barrier of height $V_{\mathrm{B}}$ at $R=R_{\mathrm{B}}$, which were studied in [7]. Most populated states before the distance $R_{\mathrm{B}}$ of adiabatic potentials (13) are labelled $\mathrm{A}, \mathrm{B}$, and $\mathrm{C}$ in Fig. 3. Peaks of the calculated barrier distribution $D\left(E_{\text {c.m. }}\right)$ (in Fig. 2) correspond to them, and there is a satisfactory agreement between the experimental and calculated distributions for the reaction ${ }^{40} \mathrm{Ca}+{ }^{90} \mathrm{Zr}$ in the number of peaks, their relative heights, and the curve shape.

\section{The neutron transfer channels coupling}

Neutron transfer channels coupled equations based on the perturbed stationary states method [1] and similar to (1) were proposed in work $[8,9]$

$$
\begin{aligned}
y_{L,} \quad \frac{L(L+1)}{R^{2}} y_{L,}+\frac{2}{\hbar^{2}}[E(R) \quad & V(R)] y_{L,}= \\
& =F_{t} \sum T(R) y_{L,} .
\end{aligned}
$$

Here $E=E_{\text {c.m. }}+Q(R), \quad Q(R)={ }_{0} \quad(R) \quad$ is the distance dependent $Q$-value, ${ }_{0}=\quad$ （ ） is the energy of the initial neutron state in the distant nucleus, $(R)$ is the two-center (molecular) energy level and $T(R)$ is the reduced kinetic energy coupling matrix

$$
T \quad(R)=\int \bar{L}_{R}^{*}(\mathbf{r}, R) \frac{-}{R} \quad(\mathbf{r}, R) d \mathbf{r} .
$$

In the simplest approximation coupling matrix elements $T(R)=T^{*}(R)$ have nonzero values only for states , appurtenant to different nuclei in the limit $R$

The coupling strength (the renormalized factor) $F_{t}>1$ is used for compensating the deletion of some complicated expressions in the exact equations of the perturbed stationary states method. Wave functions $\quad(\mathbf{r}, R)$ and energies $(R)$ of the valence neutron with the mass $m$ may be calculated in the two-center shell model by solving a stationary Schrödinger equation with the potential $U$ and the spin-orbital interaction $U_{L S}$

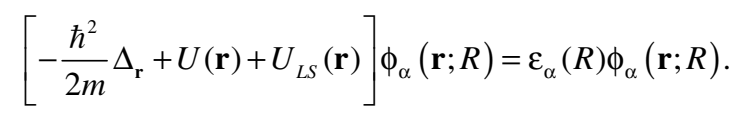

The equation (16) was solved by the method based on the series expansion of Bessel functions [7-10]. The total angular momentum projection $=1 / 2,3 / 2, \ldots$ onto the axis connecting the centers of colliding nuclei (the internuclear axis) is the quantum number of two-center states

$\left.(R)={ }_{n},(R), \quad{ }_{n}, \quad, T=T_{m n}^{()}=T_{n m}^{(}\right)$. Energies of some two-center (molecular) states in the ${ }^{18} \mathrm{O}+{ }^{58} \mathrm{Ni}$ system are plotted in Fig. 4.

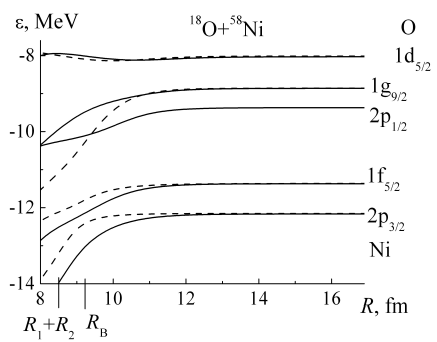

Figure 4. Energies ${ }_{n},(R)$ of two-center (molecular) states of the valence neutron with angular momentum projections onto the inter-nuclear axis $\Omega=1 / 2$ (full curves) and $\Omega=3 / 2$ (dashed curves) in the ${ }^{18} \mathrm{O}+{ }^{58} \mathrm{Ni}$ system versus the nucleus-nucleus distance $R$; scale labels: $R_{\mathrm{B}}$ is the radius of the barrier; $R_{1}$ and $R_{2}$ are radii of nuclei. The notation for states in the separated nuclei ${ }^{18} \mathrm{O}$ and ${ }^{58} \mathrm{Ni}$ is indicated.

Neutron transfers from the initial state $1 d_{5 / 2}$ of ${ }^{18} \mathrm{O}$ to unoccupied levels $1 g_{9 / 2}, 2 p_{1 / 2}, 1 f_{5 / 2}$ and partially occupied level $2 p_{3 / 2}$ of ${ }^{58} \mathrm{Ni}$ may lead to an increase in the fusion probability in the reaction ${ }^{18} \mathrm{O}+{ }^{58} \mathrm{Ni}$. Probability densities $\left.\left.\right|_{n,}\right|^{2}$ for two-center neutron wave functions changed into $1 d_{5 / 2}\left({ }^{18} \mathrm{O}\right)$ and $1 g_{9 / 2}\left({ }^{58} \mathrm{Ni}\right)$ wave functions in the limit $R \quad$ bear a similarity and strongly overlap (see Fig. 5). It leads to a non small value of the coupling matrix element $T$. Coupling channel 
matrix elements $T_{0 n}(R)$ (see Fig. 6) are similar to macroscopic coupling form factors used in CCFULL [2].
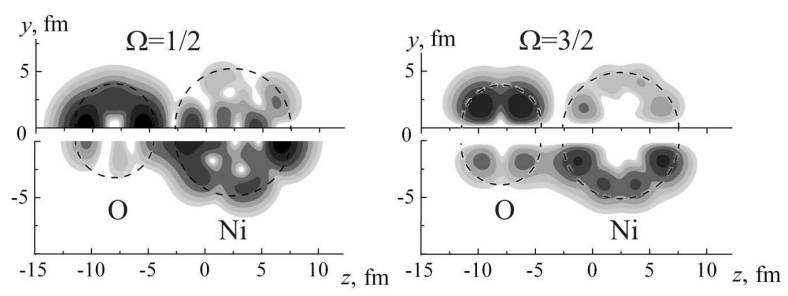

Figure 5. The probability density $\left.\right|_{n,}\left(\left.\begin{array}{ll}x=0, y, z, R & R_{\mathrm{B}}\end{array}\right|^{2}\right.$ for two-center neutron wave functions, changed into $1 d_{5 / 2}\left({ }^{18} \mathrm{O}\right.$ ) (on top) and $1 g_{9 / 2}\left({ }^{58} \mathrm{Ni}\right.$ ) (on bottom) states in the limit $R$, for the total angular momentum projection on the internuclear axis $\Omega=1 / 2$ and $\Omega=3 / 2$, circles radii equal to radii of nuclei.
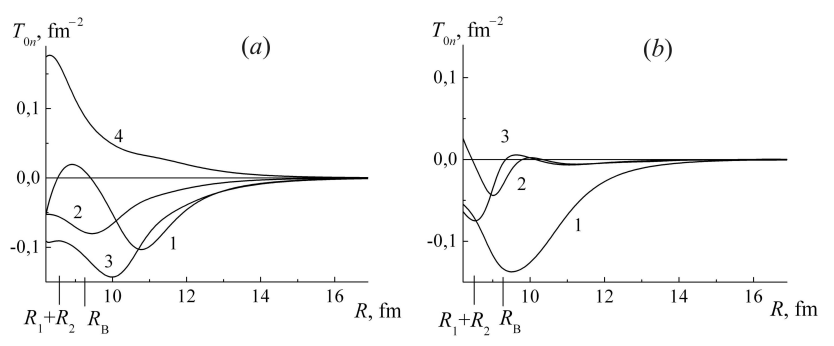

Figure 6. Coupling channel matrix elements $T_{0 n}(R)$ (15) for neutron states $1 d_{5 / 2}$ of ${ }^{18} \mathrm{O}$ (0) and ${ }^{58} \mathrm{Ni}$ (a) $1-1 g_{9 / 2}$, $2-2 p_{1 / 2}, 3-1 f_{5 / 2}, 4-2 p_{3 / 2}$ for $\Omega=1 / 2$; (b) $1-1 g_{9 / 2}$, $2-1 f_{5 / 2}, 3-2 p_{3 / 2}$ for $\Omega=3 / 2$; indexes $n$ are indicated.

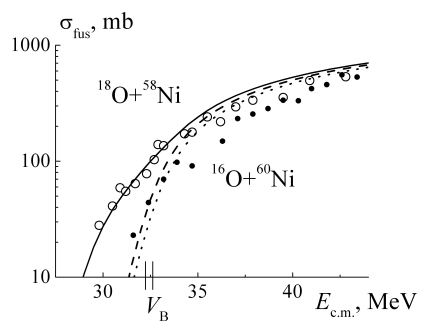

Figure 7. The experimental fusion cross section $\left(E_{\mathrm{cm}}\right)$ [11] of reactions ${ }^{18} \mathrm{O}+{ }^{58} \mathrm{Ni}$ (circles), ${ }^{16} \mathrm{O}+{ }^{60} \mathrm{Ni}$ (points) and calculation results with the proximity potential: for ${ }^{18} \mathrm{O}+{ }^{58} \mathrm{Ni}$ with the $1 d_{5 / 2}\left({ }^{18} \mathrm{O}\right)$ neutron transfer coupling (the full curve) and without this coupling (the dashed curve), for ${ }^{16} \mathrm{O}+{ }^{60} \mathrm{Ni}$ (the dotted curve), $V_{\mathrm{B}}$ is the Coulomb barrier for spherical nuclei.

Results of calculations by formulas (2), (3), (14) with the proximity potential for the fusion cross section and channel functions $y_{L, n},(R)$ are plotted in Fig. 7, 8. Near the Coulomb barrier the similarity between values of channel functions $y_{L, n}$, (Fig. 8) and coefficients $a$ of series expansion of the time-dependent valence neutron wave function $(\mathbf{r}, t) \quad[9,12,13]$ for the head-on nucleus-nucleus collision occurs for the coupling strength value $F_{t}=15$. In the vicinity of the Coulomb barrier the transition between two-center energy levels from the $1 d_{5 / 2}(\mathrm{O})$ to $1 g_{9 / 2}(\mathrm{Ni})$ with $Q(R)>0$ explains the essential increase in the fusion cross section in the reaction ${ }^{18} \mathrm{O}+{ }^{58} \mathrm{Ni}$ as compared with the reaction ${ }^{16} \mathrm{O}+{ }^{50} \mathrm{Ni}[11]$.

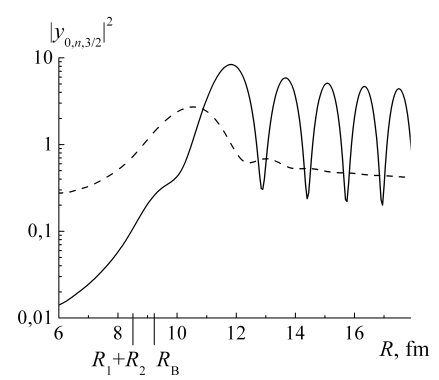

Figure 8. Channel functions $y_{L, n,}(R)$ for neutron states $1 d_{5 / 2}$ of ${ }^{18} \mathrm{O}$ (the full curve) and $1 g_{9 / 2}$ of ${ }^{58} \mathrm{Ni}$ (the dashed curve) for $E_{\text {c.m. }}=30 \mathrm{MeV}, L=0, \Omega=3 / 2$.

\section{Conclusions}

Microscopic coupled channel equations with the neutron rearrangement coupling were proposed and solved. The energy dependence of the fusion cross section in the reaction ${ }^{18} \mathrm{O}+{ }^{58} \mathrm{Ni}$ and the barrier distribution in the reaction ${ }^{40} \mathrm{Ca}+{ }^{90} \mathrm{Zr}$ have been discussed on basis of distance dependent vibration excitations energies and distance dependent $Q$-values.

\section{Acknowledgments}

This work was partially supported by the Russian Foundation for Basic Research (RFBR), research projects No 12-02-01325-a and No 13-07-00714-a.

\section{References}

1. V.P. Zhigunov and B. N. Zakhar'ev, CoupledChannel Method in Quantum Scattering Theory (Atomizdat, Moscow, 1974)

2. K. Hagino et al., Comp. Phys. Commun. 123, 143 (1999)

3. V. I. Zagrebaev and V. V. Samarin, Phys. At. Nucl. 67, 1462 (2004)

4. Nuclear Reaction Video (Nuclear Knowledge Base, FLNR, JINR, Dubna), http://nrv.jinr.ru/nrv/

5. H. Timmers, et al., Nucl. Phys. A 633, 421, (1998)

6. G. I. Marchuk, Methods of Computational Mathematics (Nauka, Moscow, 1980)

7. V. V. Samarin, Phys. of Atom. Nucl. 72, 1682 (2009)

8. V. V. Samarin, Nucl. Phys. Atom. Ener. 14, 233 (2013)

9. V. V. Samarin, Phys. of Atom. Nucl. 77, N 12 (2014) (to be published)

10. V. V. Samarin, Phys. of Atom. Nucl. 73, 1416 (2010)

11. M. Borges et al., Phys. Rev. C 46, 2360 (1992)

12. V. I. Zagrebaev, V. V. Samarin, W. Greiner. Phys. Rev. C 75, 035809 (2007)

13. V. V. Samarin, K. V. Samarin, Bull. Russ. Acad. Sci. Phys. 75, 964 (2011) 